Check for updates

Cite this: J. Mater. Chem. B, 2021 9, 3606

\author{
Guoping Chen id ab
}

\title{
Journal of Materials Chemistry B and Biomaterials Science Editor's choice web collection: "Recent advances in microfluidics"
}

\author{
DOI: $10.1039 / d 1 t b 90057 a$
}

rsc.li/materials-b

Microfluidics uses fluidic micro-channels to manipulate multiple fluids at the microscale. ${ }^{1}$ Because of the unique miniaturization features, microfluidics provides numerous advantages over conventional techniques. Microfluidics and microfluidic devices have been broadly used in biomedical fields ranging from drug delivery, microcarriers, diagnosis, biosensing, bioimaging, organs-on-a-chip, cell separation and cell encapsulation. ${ }^{2}$ This Editor's

\footnotetext{
${ }^{a}$ Research Center for Functional Materials, National Institute for Materials Science, Tsukuba, Ibaraki, 305-0044, Japan.E-mail: Guoping.CHEN@nims.go.jp ${ }^{b}$ Department of Materials Science and Engineering, Graduate School of Pure and Applied Sciences, University of Tsukuba, Tsukuba, Ibaraki, 305-8577, Japan
}

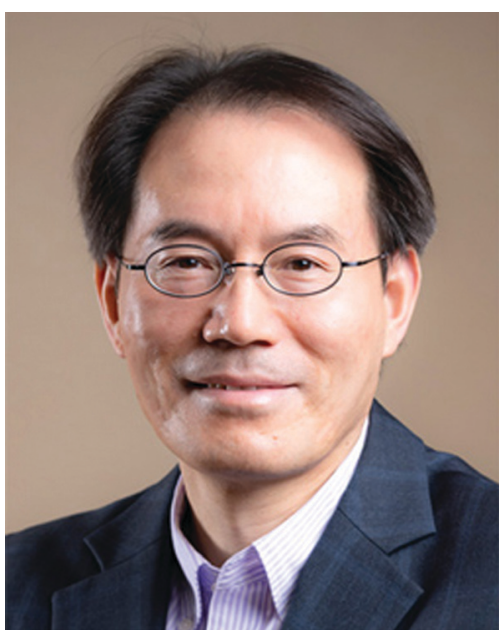

Guoping Chen collection introduces some microfluidicsrelated reviews and research articles that have recently been published in Journal of Materials Chemistry $B$ and Biomaterials Science. Journal of Materials Chemistry $B$ publishes work on the properties and synthesis of materials with applications in biology and medicine, with a particular focus on advances in materials chemistry. Biomaterials Science emphasizes work that provides insight into the underlying science and performance of biomaterials within a biological environment. This collection covers the preparation of microbeads, organs-on-a-chip, cell encapsulation, biosensors and biological assays.

One of the primary applications of microfluidics is the preparation of micro and nanomaterials, because this technology can precisely control the flow volume of multiple fluids. ${ }^{3}$ Zhang et al. summarized the latest achievements in microfluidic reactors for the controllable synthesis of $\mathrm{ZnO}$ structures and their applications in biosensing, biological separation and molecular catalysis (DOI: 10.1039/c9bm01787a). Santos et al. reported the optimized and continuous "all-inone" two-step microfluidic production of a nano-in-micro composite system (DOI: 10.1039/d0bm00743a). The system was used to prepare a pH-responsive mucoadhesive cell-mimicking system for insulin delivery. Insulin was encapsulated into PEGylated liposomes which were coated with chitosan. Yang et al. prepared
PLGA/chitosan-heparin composite microparticles with highly monodisperse sizes and a shell-core structure of a PLGA encapsulated chitosan-heparin complex by the combination of double emulsion and microfluidics (DOI: 10.1039/d0tb01593h). The composite microparticles could provide a neutral microenvironment and reduced the burst release of loaded drugs for the construction of aggregates of human mesenchymal stem cells. A combined methodology based on the merging of microfluidics and supramolecular selfassembly was established to prepare nanobowl-like structures of a single amino acid (DOI: 10.1039/d0bm01386b). The nanobowls were infused into a vesicular shell to prepare the $\mathrm{pH}$-responsive core-shell like microstructures for the delivery of anticancer drugs. Injectable pH-degradable polyvinyl alcohol microgels encapsulated with oncolytic adenovirus (OA) were prepared for localized OA delivery and cancer treatments (DOI: 10.1039/d0bm00172d). A microfluidic approach was developed to enable the high-throughput fabrication of microgels of in situ adjustable diameters, stiffness, degradability and biomolecular functionalization (DOI: 10.1039/c9bm01009b). This method enabled the integration of cell-laden microgels within cell-laden bulk hydrogel matrices to provide thoroughly tunable "microgel-in-gel" systems for creation of multiphasic in vitro models. 
Cell-laden microfluidic devices, known as organs-on-a-chip, have many advantages over conventional cell culture methods because they can provide cells with a more physiologically relevant environment. ${ }^{4}$ They can provide the cells with continuous nutrient supply and waste removal gradients, mimicking physiological flow and different factors. 3D extracellular matrices and scaffolding materials can be integrated in microfluidic devices to provide biomimetic microenvironments for cell culture. Chen et al. summarized the details of organs-on-a-chip by focusing on the latest developments and future perspectives in using scaffolding hydrogels and electrospun fibers for $3 \mathrm{D}$ cell culture in microfluidics (DOI: 10.1039/d0tb00718h). Luo et al. studied the influence of glutaraldehyde crosslinking on freestanding and semipermeable chitosan membranes assembled with flow in single-layer microfluidic networks (DOI: 10.1039/c9tb02527h). The glutaraldehyde-crosslinked chitosan membrane showed improved properties for broad applications. A microfluidic system was used as a bioreactor to generate a concentration gradient of the induction medium to control the differentiation of rat bone marrow-derived stem cells and tendon stem cells to osteocytes and tenocytes, respectively, on a decellularized tendon scaffold (DOI: 10.1039/d0bm00229a). Implantation in a tendon defect rat model indicated that incubation with the concentration gradient in the microfluidic system showed the best repair outcome.

The encapsulation of cells into microgels is attractive for applications in tissue regeneration. A microfluidic in situ microencapsulation system was used for the encapsulation of human fetal chondroprogenitor cells, articular chondrocytes and mesenchymal stem cells to investigate the chondrogenic phenotype of the microencapsulated cells in a biocompatible, assembled microgel system vs. bulk gels (DOI: 10.1039/c9bm01524h). An oilfree droplet microfluidic system was used for the one-step synthesis of composite hydrogel capsules composed of a fibrin hydrogel core and an alginate-chitosan composite shell, which enabled the formation of liver organoids from human induced pluripotent stem cells (DOI: 10.1039/d0bm01085e).

Functional nucleic acids are promising recognition elements for biosensors because they can be easily synthesized and modified. They also have the advantages of high affinity and recognition specificity, small size and high stability. Functional nucleic acids have been used as recognition elements to construct microfluidic paper-based analytical devices. Li et al. reviewed the advantages of nucleic acid-incorporated paper sensors and their applications in dot blots, lateral flow assays and microfluidic paperbased analytical devices (DOI: 10.1039/ c9tb02584g). Jeroen et al. reported a 3D DNA origami-based bioreceptor immobilized on the surface of disc-shaped microparticles in a continuous microfluidic environment (DOI: 10.1039/c9tb02439e). This method enabled the formation of a less densely packed surface with reduced steric hindrance and favored upward orientation on the sensor surface, which improved the biomolecular interactions at the sensing surface and increased the overall performance of the biosensing devices.

Microfluidics is also useful technology for biosensing and biological assays. In a review summarizing the latest advances of bioassays and biosensors for the detection of matrix metalloproteinases, microfluidics is highlighted as one of the multiplex and high-throughput analysis approaches (DOI: 10.1039/c9tb02189b). The applications of microfluidic devices in cell migration assays are highlighted in a review summarizing the current status of microphysiological systems and supporting technologies for modelling and studying wound healing (DOI: 10.1039/ d0tb00544d). Mestres et al. developed microfluidic chips integrating medical grade titanium $\left(\mathrm{Ti}_{6} \mathrm{Al}_{4} \mathrm{~V}\right)$ and used them to investigate the biological properties of titanium under dynamic conditions (DOI: 10.1039/d0bm00964d). The chips may serve as more complex in vitro platforms to offer a more reliable option to screen the biological properties of biomaterials.
Ni et al. fabricated a sensitive fluorescent microfluidic sensor based on carbon quantum dots, a cadmium telluride quantum dot aerogel and glucose oxidase for urinal glucose detection (DOI: 10.1039/ d0tb00328j). The sensor showed excellent selectivity, specificity and stability, which may enable daily urine glucose monitoring for diabetic patients in a convenient way. Shamsi et al. developed a novel method to characterize and discriminate trinucleotide repeat sequences by monitoring their capillary flow in the absence of an external driving force by using waxon-plastic microfluidics (DOI: 10.1039/ c9tb02208b). In addition, Pei et al. reviewed the progress of the detection and release of circulating tumor cells (CTC) through aptamer-functionalized nanostructured interfaces and highlighted aptamermodified microfluidic platforms for CTC capture (DOI: 10.1039/c9tb02457c). A precise flow-controlled microfluidic system with specific custom-designed chambers was fabricated to assess the combined effect of shear stress and topography on the adhesion, orientation and elongation of Schwann cells (DOI: 10.1039/d0bm01218a). The system could simulate the mechanical stimuli and topography of in vivo environments.

This collection only lists some of the many recent articles published in Journal of Materials Chemistry $B$ and Biomaterials Science on the topic of microfluidics. I hope the collection is informational and useful for our readers.

\section{References}

1 G. M. Whitesides, Nature, 2006, 442, 368-373.

2 E. K. Sackmann, A. L. Fulton and D. J. Beebe, Nature, 2014, 507, 181-189.

3 W. Li, L. Zhang, X. Ge, B. Xu, W. Zhang, L. Qu, C. Choi, J. Xu, A. Zhang, H. Lee and D. A. Weitz, Chem. Soc. Rev., 2018, 47, 5646-5683.

4 A. Polini, L. Prodanov, N. S. Bhise, V. Manoharan, M. R. Dokmeci and A. Khademhosseini, Expert Opin. Drug Discovery, 2014, 9, 335-352. 